\title{
Pesquisa exploratória qualitativa sobre representação técnica de projetos de interiores
}

Exploratory qualitative research on technical representation of interior projects

SILVA, Arabella Natal Galvão da; Doutoranda; UNICURITIBA e UFPR

arabella.n.galvao@gmail.com

GONÇALVES, Laisy Esther; Graduanda; UNICURITIBA

laisyesther@gmail.com

BACINELLO, Paula M escko; Graduanda; UNICURITIBA

paulabacinello@hotmail.com

\section{Resumo}

O projeto de móveis sob medida necessita ser representado com clareza, pois dificuldades de entendimento causam diversos prejuízos. A representação técnica de projetos de móveis é o tema do projeto de iniciação científica do qual fazem parte as autoras. Este artigo se constitui como parte dos trabalhos e teve como objetivo identificar referencial teórico sobre a representação técnica de projetos de interiores. O método adotado foi a Revisão Bibliográfica, Sistemática e Assistemática. Os resultados sugerem a carência de referencial teórico sobre o tema.

Palavras Chave: representação gráfica; desenho técnico; design de interiores.

\begin{abstract}
The furniture design custom-made needs to be represented clearly, because of difficulties understanding cause many losses. The technical representation of furniture designs is the research project theme which includes the authors. This article represents part of the work and aims to identify theoretical framework on the technical representation of interior designs. The method adopted was the Literature Review, systematic and unsystematic. The results suggest the lack of theoretical framework on the subject.
\end{abstract}

Keywords: graphic representation; technical drawing; interior design. 


\section{plural $^{\text {GAI }}$}

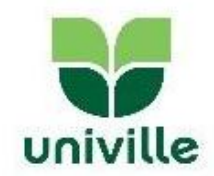

\section{Introdução}

O Design de Interiores tem se consolidado como profissão independente da Arquitetura nos últimos anos. A demanda pelos serviços do designer de interiores tem aumentado, por motivos diversos. Iwata et al. (2003) afirmam que a demanda é maior em razão da falta de segurança das grandes cidades, que faz com que as pessoas prefiram realizar atividades em casa ou outros ambientes fechados, impulsionando a busca por um profissional que torne tais espaços agradáveis e confortáveis. Além disso, o poder aquisitivo da classe média tem aumentado, ampliando o mercado deste profissional que era restrito às camadas socioeconômicas mais altas. Tais dados revelam a importância social e econômica deste profissional.

O projeto de móveis tem grande peso dentre os projetos executados pelo designer de interiores, conforme afirmam Pita e Costa Filho (2012): "o mobiliário é um fator crucial num projeto de interiores, pois ele define e dá identidade ao espaço, através de formas, cores, superfícies e arranjos". Na marcenaria sob medida, os móveis têm de ser representados dentro do espaço arquitetônico, para facilitar a compreensão da relação entre o móvel e a arquitetura. Ou seja, não é possível representar graficamente o móvel isolado das paredes, piso e teto, pois as dimensões e a estrutura do móvel vão depender desses elementos. No entanto, não existe uma norma de desenho que oriente essa representação em específico. As normas existentes tratam apenas da arquitetura ou apenas do objeto isolado de seu contexto. Os profissionais e professores da área utilizam as referências da arquitetura e do design de produto para subsidiar as decisões de projeto, além da própria experiência de cada profissional. Porém não há uma padronização, ou seja, o uso que se faz das normas de desenho existentes depende da interpretação de cada profissional, o que resulta em formas de representação distintas umas das outras, podendo gerar dúvidas no marceneiro.

Este contexto evidencia a necessidade de intensificação das pesquisas em design de interiores, mais especificamente no projeto de mobiliário. Desta forma, este artigo tem como objetivo identificar referencial teórico sobre a representação técnica de projetos de interiores, como subsídio ao projeto de iniciação científica do qual fazem parte as autoras. O método adotado é a Revisão Bibliográfica Sistemática e Assistemática, sendo as buscas realizadas no portal de periódicos da Capes, no portal INFOHAB - Centro de Referência e Informação em Habitação e nos anais das últimas edições do P\&D Design - Congresso Brasileiro de Pesquisa e Desenvolvimento em Design.

Os resultados reforçam a carência de publicações nesta área, além de evidenciar a relevância do projeto de iniciação científica em andamento, que buscará respostas para a seguinte questão: qual a melhor maneira de representar graficamente o móvel sob medida, de modo que o marceneiro compreenda perfeitamente o que deverá ser executado?

\section{Método}

Para identificar referencial teórico sobre a representação gráfica de projetos de interiores foi realizada uma Revisão Bibliográfica Sistemática - RBS. A RBS baseia-se na definição de estratégias e métodos sistematizados para realizar buscas de referenciais teóricos 


\section{GAMPI
plural ${ }^{15}$}

e analisar os resultados encontrados, de modo que seja possível repetir o processo por meio de "ciclos contínuos até que os objetivos da revisão sejam alcançados" (CONFORTO; AMARAL; SILVA, 2011, p. 2). O protocolo definido previamente para a realização as buscas é o seguinte: (1) busca de artigos por meio de palavras-chave; (2) leitura dos títulos dos artigos encontrados; (3) leitura dos resumos dos artigos selecionados a partir dos títulos; (4) leitura da introdução e da conclusão dos artigos selecionados a partir dos resumos; (5) leitura integral dos artigos selecionados a partir da introdução e da conclusão.

A RBS que gerou os resultados descritos neste artigo foi desenvolvida em três frentes. $\mathrm{Na}$ primeira frente foi feita uma busca no portal de periódicos da Coordenação de Aperfeiçoamento de Pessoal de Nível Superior (CAPES) com vistas a encontrar artigos internacionais sobre o tema. No Quadro 1 podem ser observados os strings buscados, os filtros empregados e o número de artigos encontrados.

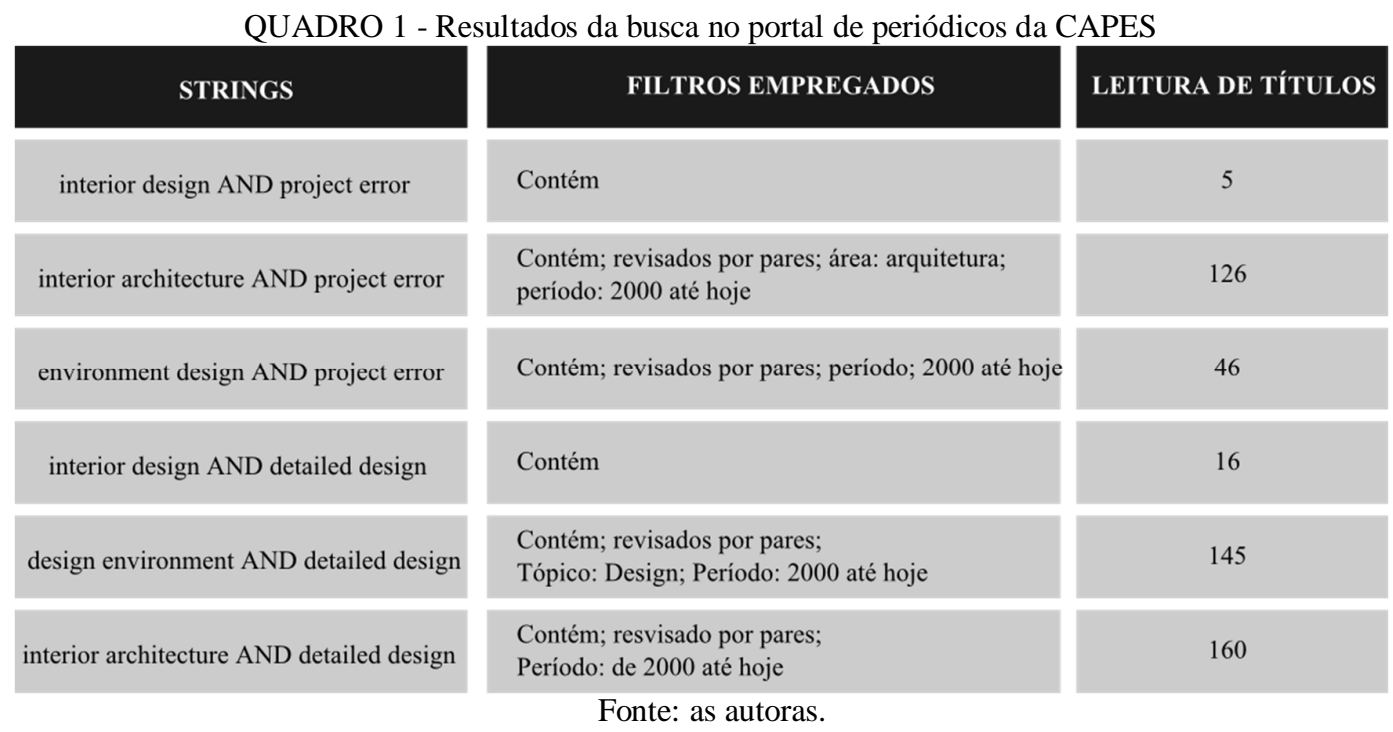

$\mathrm{Na}$ segunda frente foi realizada busca nos anais do P\&D Design. Sendo este o maior congresso de design brasileiro, esperava-se encontrar artigos recentes de pesquisadores brasileiros sobre o tema de pesquisa. A busca foi feita nos anais dos eventos que ocorreram em 2014 e em 2012, que são as duas últimas edições do congresso até a redação do presente artigo. Nesse caso, a busca por palavras-chave é mais difícil, pois nem sempre os anais estão disponibilizados em bancos de dados com mecanismos de busca. Desta forma, procedeu-se a leitura de todos os títulos publicados no P\&D 2014, buscando atender ao objetivo desta pesquisa, tendo em vista que os anais foram publicados num repositório acessado pela internet. Os anais do P\&D 2012 foram acessados em DVDs. Desta forma foi possível identificar pastas por áreas, sendo selecionadas as pastas sobre design de ambientes. Ainda assim, foi feita a leitura de todos os títulos dos artigos presentes nestas pastas. Após a leitura de títulos, seguiram-se os mesmos passos descritos no protocolo de pesquisa.

A terceira frente foi a busca no Centro de Referência e Informação em Habitação (INFOHAB). O INFOHAB é um portal "pensado e implementado para aprimorar os mecanismos de sistematização e socialização da informação na área do ambiente construído" 


\section{GAMPI plural 15}

(ANTAC, 2015). Por meio do portal, é possível realizar buscas por palavras-chave, o que foi feito conforme o Quadro 2.

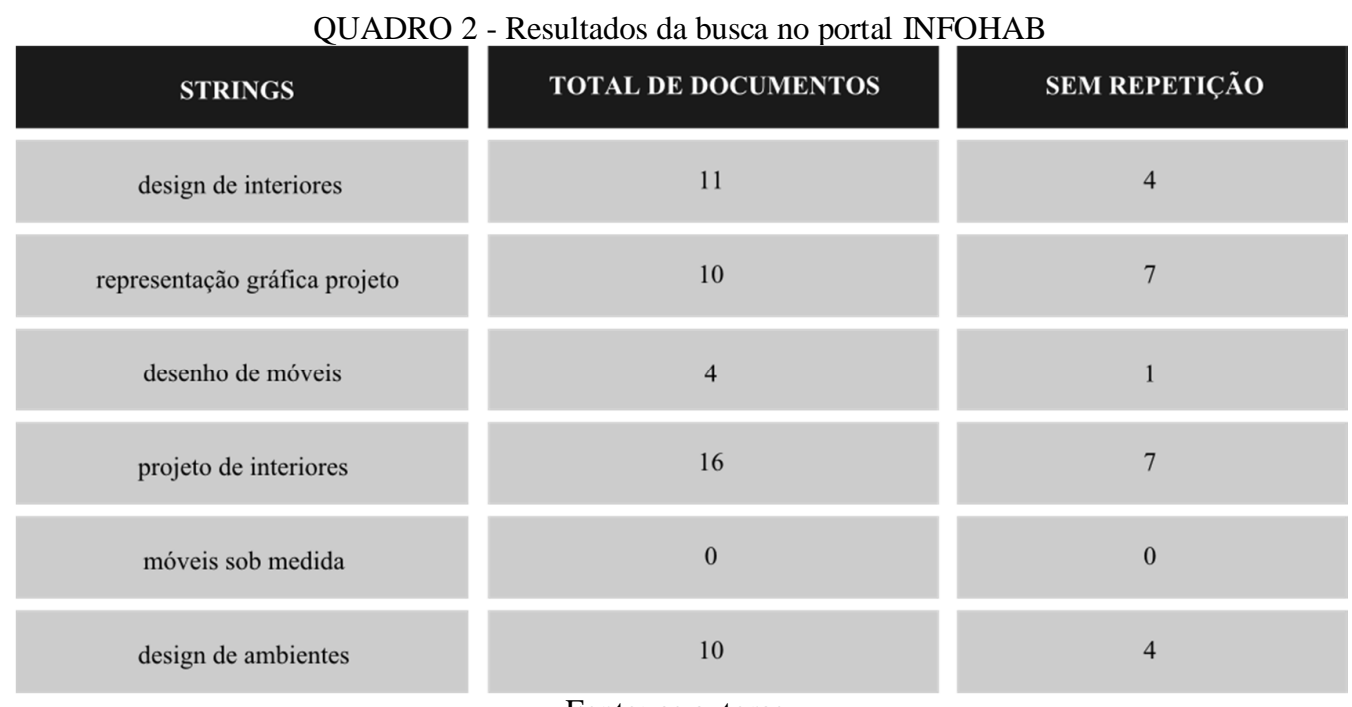

Fonte: as autoras.

As buscas foram complementadas por uma Revisão Bibliográfica Assitemática (RBA) feita em anais de outros eventos relacionados ao tema, como o Simpósio Nacional de Geometria Descritiva e Desenho Técnico (GRAPHICA), na base de dados Scielo e no mecanismo de buscas na internet Google.

\section{Resultados}

A partir da pesquisa descrita no tópico anterior, foram selecionados os artigos para leitura completa, conforme o Quadro 3, a seguir.

A busca realizada nos anais do Congresso P\&D Design não trouxe nenhum artigo relevante para o tema desta pesquisa.

\section{Discussão}

Os resultados encontrados sugerem a inexistência de estudos relacionados à representação técnica de projetos de interiores. Além disso, os resultados evidenciam a ênfase de pesquisas voltadas para o ambiente acadêmico e para a representação gráfica de apresentação dos projetos.

QUADRO 3 - Artigos selecionados para leitura completa 


\section{GAMPI}
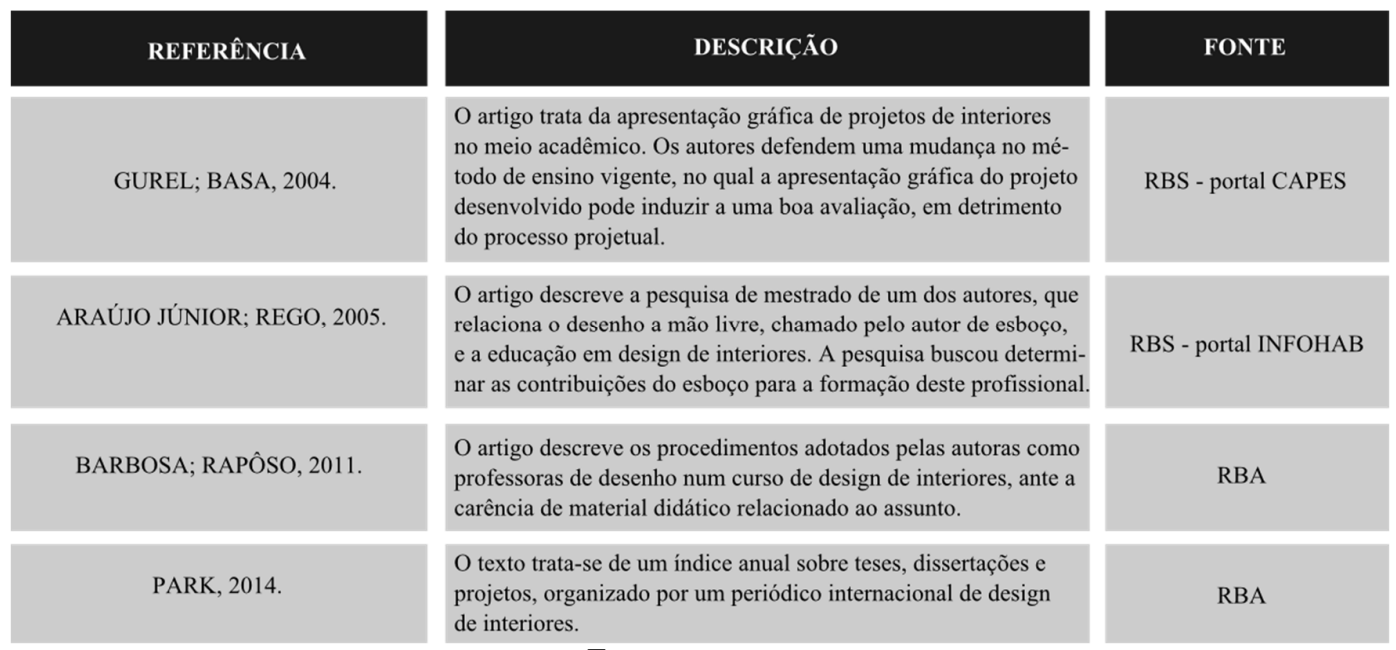

Fonte: as autoras.

Os dois artigos nacionais encontrados tratam de pesquisas em cursos superiores de design de interiores. No primeiro deles, Araújo Júnior e Rego (2005) abordam o esboço, definindo qual forma de desenho pode ser assim considerado e ressaltando sua importância para o desenvolvimento da habilidade do desenho durante a graduação de Design de Interiores. $\mathrm{O}$ artigo apresenta cinco das percepções necessárias apresentadas nos estudos de Edwards (2000) para a execução de desenhos de observação. Baseando-se nestas percepções os autores analisam o desenvolvimento de alunas durante o período letivo, onde as habilidades de desenho eram observadas de acordo com a didática das disciplinas de expressão gráfica. Desta forma Araújo Júnior e Rego (2005) concluíram que o desenho a mão livre tem grande importância no desenvolvimento de profissionais da área de Design de Interiores, pois por meio dele é possível adquirir ou aprimorar a capacidade do desenho em um período consideravelmente curto.

Barbosa e Rapôso (2011) comentam a carência de materiais didáticos voltados para a área de design de interiores e evidenciam a importância da apresentação gráfica de projetos. Um projeto bem representado graficamente facilita a compreensão pelo cliente e ajuda na sua boa aceitação (Barbosa; Rapôso 2011). As autoras relatam as técnicas de desenho e pintura rápidas e de fácil aplicação, verificando o que cada material disponível pode oferecer para uma melhor visualização da proposta. Além disso, procuram auxiliar os estudantes em seus projetos utilizando materiais de custo reduzido, pois grande parte destes não investe em bons materiais artísticos. As técnicas favorecem o aprendizado dos estudantes, já que não há materiais didáticos sobre desenho e representação gráfica para iniciantes. Após a pesquisa realizada, Barbosa e Rapôso (2011) concluem que a carência de materiais didáticos voltados para a área impossibilita os alunos a aprenderem mais sobre desenhos técnicos e projetos gráficos, bem como os livros existentes são voltados para profissionais da área e não para iniciantes. Finalmente, o custo elevado dos materiais artísticos dificulta seu uso por estudantes de design de interiores para elaborar um bom projeto gráfico, sendo necessário desenvolver as técnicas com materiais mais simples e acessíveis.

Gurel e Basa (2004) concordam que a apresentação gráfica dos projetos é importante para sua perfeita finalização. No entanto, os autores fazem fortes críticas à valorização dada a 


\section{GAMPI}

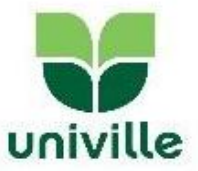

esta etapa, o que sugere uma semelhança entre as práticas acadêmicas internacionais (especialmente na Turquia, onde o estudo foi realizado) e brasileiras, com base nos artigos lidos integralmente. Os autores destacam que o poder da expressão estética de um projeto torna-se um fator dominante na percepção que se tem do próprio projeto. Por consequência, isso pode acabar interferindo sobremaneira o processo educacional, tirando o foco do desenvolvimento da solução projetual (GUREL; BASA, 2004). Após a pesquisa realizada, Gurel e Basa (2004) concluem que tanto as referências bibliográficas sobre apresentação gráfica, como os alunos e professores do curso pesquisado concordam que há uma grande valorização da apresentação gráfica dos projetos desenvolvidos por estudantes. No entanto, os autores mantém sua crítica inicial, de que esta valorização é excessiva, com base na sua percepção pessoal e naquilo que o meio profissional demonstra ser importante.

O texto de Park (2014) aborda uma lista de teses, dissertações e projetos da área de design de interiores. A pesquisa é realizada anualmente pelo Journal of Interior Design e lista o resultado das pesquisas de pós-graduação realizadas na área, nos Estados Unidos, durante o ano anterior. Nesta edição foram listadas 9 dissertações (equivalentes às teses de doutorado no Brasil), 123 teses (equivalentes às dissertações de mestrado no Brasil) e 82 projetos criativos (projetos de mestrado em andamento). Os títulos foram classificados por tema, para facilitar a análise, e os projetos em andamento não foram considerados. Dentre as teses de doutorado, o tema mais abordado foi a sustentabilidade (3 teses) e aspectos culturais dos ambientes (2 teses). Em relação às dissertações de mestrado, 37 delas relacionam-se a temas ligados à saúde e ao bem-estar e 33 relacionam-se aos aspectos culturais dos espaços, sendo estes os temas mais recorrentes. A partir da leitura dos títulos das teses e dissertações, não foi possível identificar nenhuma que tivesse relação com o tema deste artigo.

Os artigos lidos abordam assuntos relevantes para a pesquisa em design, não só em ambiente acadêmico, mas também no meio profissional. A lacuna observada em relação ao tema de investigação deste artigo sugere que há uma oportunidade de pesquisa a ser explorada, ainda que se possa ampliar as buscas de referenciais teóricos.

\section{Conclusão}

Os serviços do designer de interiores têm sido demandados pela sociedade com maior intensidade nos últimos anos. Isso justifica a ampliação de pesquisas nesta área, haja vista a percepção recente de que esta é uma profissão independente da arquitetura. Dentre os projetos desenvolvidos pelo designer de interiores, o de móveis ganha importância pelo impacto que causa ao espaço e pelos prejuízos que causa quando é executado incorretamente ou é mal compreendido pelo executor do serviço. Desta forma, este artigo teve como objetivo identificar referencial teórico sobre a representação técnica de projetos de interiores, como subsídio ao projeto de iniciação científica do qual fazem parte as autoras. O método adotado foi a Revisão Bibliográfica, realizada de modo sistemático e assistemático.

Os resultados encontrados sugerem a inexistência de pesquisas e artigos sobre a representação técnica de móveis sob medida. Foram identificados artigos, nacionais e internacionais, que tratam da apresentação gráfica do projeto, com ênfase nos aspectos estéticos e ilustrativos. No entanto, não foi encontrado nenhum artigo que trate da representação técnica do projeto, que é utilizada pelo marceneiro para a fabricação dos móveis. Tal fato pode sugerir que esta representação está resolvida pelas normas de desenho técnico existentes, porém a pesquisa de iniciação científica em andamento mostra resultados 5o GAM PI Plural, 2015, UNIVILLE, Joinville, SC 


\section{GAMPI}

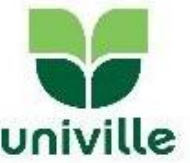

diferentes, que serão apresentados em artigo posterior. Além disso, os artigos encontrados referem-se sempre à abordagens acadêmicas, ou seja, o ensino dos futuros profissionais de design de interiores em relação a apresentação gráfica dos projetos. Observa-se, neste caso, uma oportunidade de pesquisa no meio profissional, para verificar a percepção da área em relação a esta apresentação gráfica.

Por fim, esta pesquisa mostra possibilidades de novos estudos na área de design de interiores, especialmente em relação à representação técnica dos projetos. Contribuições neste sentido poderão auxiliar o desenvolvimento do campo teórico do design de interiores no Brasil.

\section{Referências}

ANTAC - Associação Nacional de Tecnologia do Ambiente Construído. Sobre o INFOHAB - Apresentação. Disponível em: <http://www.infohab.org.br/index/institucional > Acesso em: 23 jul. 2015.

ARAÚJO JÚNIOR, Aarão Pereira de; REGO, Rogéria Gaudêncio do. Desenvolvendo a Habilidade para o Desenho de Esboço no Curso Superior de Tecnologia em Design de Interiores do CEFET-PB. In: Revista Princípia, João Pessoa, n.12, p. 6-14, 2005.

BARBOSA, Gabriela Biana; RAPÔSO, Áurea. Técnicas de Apresentação Gráfica para o Design de Interiores: experiência alagoana. In: Simpósio Nacional de Geometria Descritiva e Desenho Técnico - GRAPHICA, 20., Rio de Janeiro, 2011. Anais... Rio de Janeiro: UFRJ, 2011.

CONFORTO, E.C,; AMARAL, D. C.; SILVA, S. L. Roteiro para revisão bibliográfica sistemática: aplicação no desenvolvimento de produtos e gerenciamento de projetos. In: CONGRESSO BRASILEIRO DE GESTÃO DE DESENVOLVIMENTO DE PRODUTO CBGDP, 8., Porto Alegre, 2011. Anais... Porto Alegre: UFRGS, 2011. p. 1-12.

GUREL, Meltem O.; BASA, Inci. The Status of Graphical Presentation in Interior/Architectural Design Education. In: International Journal of Art and Design Education, vol.2, n. 2, p.192-206, 2004.

IWATA, Nara; ROCHA, Ana Beatriz; SALEIRO FILHO, Mario. Interiores exteriorizados / Exteriores interiorizados - $\mathrm{O}$ ensino do projeto de arquitetura de interiores. In: Seminário Nacional sobre Ensino e Pesquisa em Projeto de Arquitetura - PROJETAR, 1., Natal, 2013. Anais... Natal: PPGAU-UFRN, 2003.

PARK, Nam-Kyu. Annual index of Interior Design dissertations, theses, and creative projects. In: Journal of. Interior Design, v. 39, n. 4, p. 33-42, 2014.

PITA, Camila Afonso Ferreira; COSTA FILHO, Lourival Lopes. Atribuições dos arquitetos e 

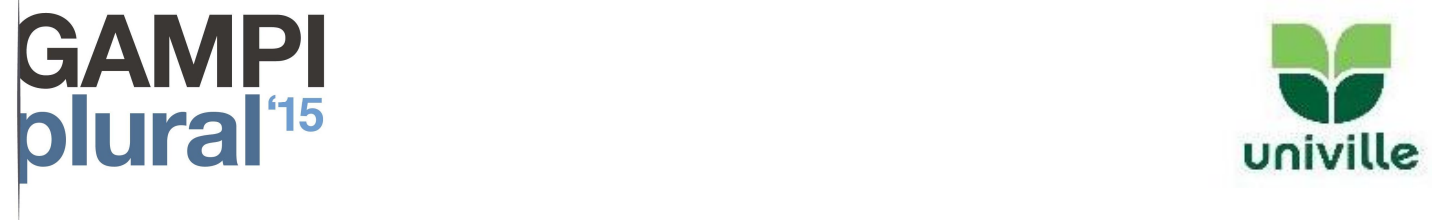

designers que atuam no projeto de interiores. In: Congresso Brasileiro de Pesquisa e

Desenvolvimento em Design - P\&D, 10., São Luís, 2012. Anais... São Luís: UFMA, 2012. 